\title{
Spontaneous Emergence of Physical Structures and Observable Formations: Fluctuations, Waves, Turbulent Pulsations and So on
}

\author{
L. I. Petrova \\ Department of Computational Mathematics and Cybernetics, Moscow State University, Moscow, Russia \\ Email: ptr@cs.msu.su
}

Received 26 February 2016; accepted 20 May 2016; published 23 May 2016

Copyright ( 2016 by author and Scientific Research Publishing Inc.

This work is licensed under the Creative Commons Attribution International License (CC BY).

http://creativecommons.org/licenses/by/4.0/

(c) (i) Open Access

\begin{abstract}
As it is known, the closed inexact exterior form and associated closed dual form make up a differential-geometrical structure. Such a differential-geometrical structure describes a physical structure, namely, a pseudostructure on which conservation laws are fulfilled (A closed dual form describes a pseudostructure. And a closed exterior form, as it is known, describes a conservative quantity, since the differential of closed form is equal to zero). It has been shown that closed inexact exterior forms, which describe physical structures, are obtained from the equations of mathematical physics. This process proceeds spontaneously under realization of any degrees of freedom of the material medium described. Such a process describes an emergence of physical structures and this is accompanied by an appearance of observed formations such as fluctuations, waves, turbulent pulsations and so on.
\end{abstract}

\section{Keywords}

Skew-Symmetric Form, Nonidentical Relation, Degenerate Transformation, the Transition from the Nonintegrable Manifolds to the Integrable Structures

\section{Introduction}

Closed inexact exterior forms and associated closed dual form describe structures which have both mathematical and physical meaning. Integral structures and physical structures, i.e. structures on which conservation laws are fulfilled, are just such structures.

How to cite this paper: Petrova, L.I. (2016) Spontaneous Emergence of Physical Structures and Observable Formations: Fluctuations, Waves, Turbulent Pulsations and So on. Journal of Applied Mathematics and Physics, 4, 863-870. 
In present paper, a mathematical and physical sense of such structures and their role in mathematical physics will be disclosed. It will be shown that the process of emerging physical structures describes evolutionary processes in material media and discloses a mechanism of emerging various observable formations (fluctuations, waves, vortices, turbulent pulsations and so on).

\section{What Are Physical Structures?}

To define what are physical structures one should note some properties of skew-symmetric forms, corresponding to the conservation laws.

Some properties of closed inexact exterior forms. The external differential form of the degree $p$ ( $p$-form) can be written as [1] [2]:

$$
\theta^{p}=\sum_{i_{1} \cdots i_{p}} a_{i_{1} \cdots i_{p}} d x^{i_{1}} \wedge d x^{i_{2}} \wedge \cdots \wedge d x^{i_{p}} \quad 0 \leq p \leq n .
$$

Here $a_{i_{1} \cdots i_{p}}$ is the function of independent variables $x^{1}, \cdots, x^{n}, n$ is the space dimension, and $d x^{i}, d x^{i} \wedge d x^{j}$, $d x^{i} \wedge d x^{j} \wedge d x^{k}$, is the local basis subject to the condition of skew-symmetry:

$$
\begin{aligned}
& d x^{i} \wedge d x^{i}=0 \\
& d x^{i} \wedge d x^{j}=-d x^{j} \wedge d x^{i} \quad i \neq j .
\end{aligned}
$$

The exterior form differential $d \theta^{p}$ is expressed by the formula

$$
d \theta^{p}=\sum_{i_{1} \cdots i_{p}} d a_{i_{1} \cdots i_{p}} \wedge d x^{i_{1}} \wedge d x^{i_{2}} \wedge \cdots \wedge d x^{i_{p}} .
$$

The form called as a closed one if its differential equals to zero:

$$
d \theta^{p}=0 .
$$

From condition (4) one can see that the closed form is a conservative quantity. This means that such a form can correspond to the conservation law (for physical fields), i.e. is a conservative quantity.

If the form be closed only on pseudostructure, i.e. this form is a closed inexact one, the closure condition can be written as

$$
d_{\pi} \theta^{p}=0 .
$$

In this case the pseudostructure $\pi$ obeys the condition

$$
d_{\pi}^{*} \theta^{p}=0
$$

here ${ }^{*} \theta^{p}$ is a dual form.

From conditions (5) and (6) one can see that the dual form (pseudostructure) and closed inexact form (conservative quantity) describe a conservative object that can also correspond to some conservation law.

Differential-geometrical structures and their physical meaning. The closed dual form and associated closed inexact exterior form made up a differential-geometrical structure. Such a differential-geometrical structure describes a conservative object, that is, a pseudostructure with conservative quantity. (A closed dual form describes a pseudostructure. And a closed exterior form describes a conservative quantity, since the differential of closed form is equal to zero).

The pseudostructure with conservative quantity is a structure on which a conservation law is fulfilled (In particular, structures, from which physical fields are formatted, are such structures. As it will be shown below, the integral structures of differential equations are such structures).

\section{Emergence of Physical Structures}

To describe the mechanism of physical structure emergence, one needs to understand how the closed exterior forms are obtained.

It turns out that closed inexact exterior forms are obtained from differential equations describing any processes. However, here there is a certain peculiarity. Closed exterior skew-symmetric forms cannot be directly 
obtained from differential equations. They are realized only in evolutionary process.

From differential equations it follows nonidentical relation that contains the skew-symmetric differential forms, whose basis is nonintegrable manifolds (as opposed to exterior skew-symmetric forms). Closed inexact exterior forms are spontaneously obtained from such skew-symmetric differential forms under realization of any degrees of freedom.

Such results are obtained when studying the integrability of differential equations that depends, firstly, on the consistency of derivatives along different directions and, secondly, on the consistency of equations in the set of equations (It should be noted that the integrability of ordinal differential equations depends on the consistency between derivatives and initial data).

Below it will be considered two types of equations: first-order partial differential equation and the equations of mathematical physics.

\subsection{Physical Structures of First-Order Partial Differential Equation}

Let us consider the first-order partial differential equation

$$
F\left(x^{i}, u, p_{i}\right)=0, \quad p_{i}=\partial u / \partial x^{i} .
$$

In order to Equation (7) be integrable, the derivatives of that equation must be consistent, that is, they must made up a differential.

Let us consider the functional relation

$$
d u=\theta
$$

where $\theta=p_{i} d x^{i}$ (the summation over repeated indices is implied). Here $\theta=p_{i} d x^{i}$ is a skew-symmetric differential form of the first degree.

If relation (8) is identical one, that is, the differential form $\theta=p_{i} d x^{i}$ is a differential (as the left-hand side of relation (8)), this relation can be integrated. This means the solution $u$ of the differential equation will be a function, that is, it will depend only on variables $x^{i}$.

The specific feature of functional relation (8) is that, in the general case, when differential Equation (7) describes any physical processes, this relation turns out to be nonidentical one.

In the general case, from Equation (7) it does not follow (explicitly) that the derivatives $p_{i}=\partial u / \partial x^{i}$, which obey to the equation (and given boundary or initial conditions of the problem), made up a differential. That is, from Equation (7) it does not follow that the differential form $\theta=p_{i} d x^{i}$ is a differential (like the left-hand side in Equation (8)).

For differential form $\theta$ be a differential, it is necessary that the mixed derivatives of the function $u$ must be permutable. That is, the commutator $K_{i j}=\partial p_{j} / \partial x^{i}-\partial p_{i} / \partial x^{j}$ of the differential form $\theta$ must vanish.

If the equations describe any processes (without any supplementary conditions), the commutator $K_{i j}$ of the differential form $\theta$ is not equal to zero. The form $\theta=p_{i} d x^{i}$ turns out to be unclosed and is not a differential like the left-hand side of relation (8). Functional relation (8) appears to be nonidentical.

This means that Equation (7) is not integrable identically. It can be integrated only at additional conditions when the derivatives of the differential equation made up a differential.

The closure condition for the form $\theta=p_{i} d x^{i}$ and that for relevant dual form (in the present case the functional $F$ plays a role of a form dual to $\theta$ ) are such additional conditions:

$$
\left\{\begin{array}{l}
d F\left(x^{i}, u, p_{i}\right)=0 \\
d\left(p_{i} d x^{i}\right)=0
\end{array} .\right.
$$

The conditions (9) are conditions for obtaining the closed inexact exterior form that is a differential (but only an interior differential on a certain structure).

If we expand the differentials, we get a set of homogeneous equations with respect to $d x^{i}$ and $d p_{i}$ (in the $2 n$-dimensional space):

$$
\left\{\begin{array}{l}
\left(\frac{\partial F}{\partial x^{i}}+\frac{\partial F}{\partial u} p_{i}\right) d x^{i}+\frac{\partial F}{\partial p_{i}} d p_{i}=0 \\
d p_{i} d x^{i}-d x^{i} d p_{i}=0
\end{array}\right.
$$


It is well-known that vanishing the determinant composed of coefficients at $d x^{i}, d p_{i}$ is a solvability condition for the system of homogeneous differential equations. This leads to relations:

$$
\frac{d x^{i}}{\partial F / \partial p_{i}}=\frac{-d p_{i}}{\partial F / \partial x^{i}+p_{i} \partial F / \partial u} .
$$

Relations (11) specify the integrating direction, namely, a pseudostructure, on which the form $\theta=p_{i} d x^{i}$ turns out to be closed one, i.e. it becomes a differential, and from relation (8) the identical relation is produced. On the pseudostructure the derivatives of differential Equation (7) constitute a differential $\delta u=p_{i} d x^{i}=d u$ (on the pseudostructure), and this means that the solution of Equation (7) becomes a function. Solutions, namely, functions on the pseudostructures formed by the integrating directions, are the so-called generalized solutions.

One can see that the pseudostructure, which is defined by relation (11) and on which the differential $d u$ (interior only on the pseudostructure) is obtained, is an integral structure. And yet the pseudostructure (dual form), which is defined by relation (11), and the differential du (closed inexact exterior form) is a physical structure since on that the conservation law is fulfilled.

[If we find the characteristics of Equation (7), it appears that the conditions (11) are equations for characteristics [3]. That is, the characteristics are examples of structures that are integral and physical ones. Here it is worth noting the fact that the realization of the characteristics (emergence of physical structure) is connected with the degenerate transformation. It occurs the transition from the initial tangent manifold to the characteristics, which appears to be a degenerate transformation, namely, the determinant of the set of Equations (10) becomes zero. The derivatives of Equation (7) are transformed from the tangent (nonintegrable) manifold to the characteristics (integral structures) that belong to the cotangent (integrable) manifold.]

(Below, when studying the integrability of the equations of mathematical physics, it will be shown that the additional conditions, which lead to emergence of physical structures, are realized spontaneously in the evolutionary process. The degenerate transformation corresponds to this. As it will be shown, such additional conditions are caused by some degrees of freedom. The vanishing of such functional expressions as determinants, Jacobians, Poisson's brackets, residues, and others corresponds to these additional conditions. In the present case, as one can see, to the additional condition it corresponds the vanishing of the determinant.)

The first-order partial differential equation has been analyzed, and the functional relation with the form of the first degree has been considered.

Similar functional properties have all differential equations describing physical processes. And, if the order of the differential equation be $k$, the functional relation with the $k$-degree form corresponds to this equation.

In a similar way the ordinary differential equation can be investigated. In this case the integrability of the equation is connected with the consistency between derivatives and initial data.

\subsection{Physical Structures of the Equations of Mathematical Physics}

While studying the integrability of partial differential equations, the consistency of derivatives with respect to different variables was analyzed by using the nonidentical functional relation. When describing physical processes in continuous media (in material systems) one obtains not one differential equation but a set of differential equations. And in this case it is necessary to investigate the consistency of not only derivatives with respect to different variables but also the consistency of the equations of this set. In this case, from this set of equations one also obtains a nonidentical relation that enables one to investigate the integrability of equations, the specific features of their solutions and describe the process of appearing physical structures.

The equations of mechanics and physics of continuous media (of material systems such as the thermodynamic, gas-dynamic and cosmic systems as well as the systems of charged particles and others) is a set of equations that describe the conservation laws for energy, linear momentum, angular momentum, and mass. The Euler and Navier-Stokes equations are examples of such a set of equations [4].

[It should be noted that the conservation laws for material systems are described by differential equations since they are balance ones (they specify the balance between the variations of physical quantities and external actions). In contrast to this, the conservation laws for physical fields are described by closed exterior forms since they are exact (point out to the availability of conserved quantities).]

Let us analyze the equations of energy and linear momentum.

In the accompanying frame of reference, which is tied to the manifold built by the trajectories of particles 
(elements) of material system (the Lagrangian reference system is an example), the equation of energy is written in the form

$$
\frac{\partial \psi}{\partial \xi^{1}}=A_{1}
$$

Here $\xi^{1}$ are the coordinates along the trajectory, $\psi$ is the functional of the state that specifies material system, $A_{1}$ is the quantity that depends on specific features of the system and on energy actions onto the system $\{$ The action functional, entropy, wave function can be regarded as examples of the functional $\psi$. Thus, the equation for energy expressed in terms of the action functional $S$ has a similar form: $\partial S / \partial \xi^{1}=D S / D t=L$, where $\psi=S$ and $A_{1}=L$ is the Lagrange function. The equation for the energy of ideal gas can be presented in the form [4]: $\partial s / \partial \xi^{1}=D s / D t=0$, where $s$ is the entropy $\}$.

In a similar way, in the accompanying frame of reference the equation for linear momentum appears to be reduced to the equation of the form

$$
\frac{\partial \psi}{\partial \xi^{v}}=A, \quad v=2, \cdots
$$

where $\xi^{v}$ are the coordinates in the direction normal to the trajectory, $A_{v}$ are the quantities that depend on the specific features of material system and on force actions.

Equations (12) and (13) can be convoluted into the relation

$$
d \psi=A_{\mu} d \xi^{\mu}, \quad(\mu=1, v)
$$

where $d \psi$ is the differential expression $d \psi=\left(\partial \psi / \partial \xi^{\mu}\right) d \xi^{\mu}$.

(In paper [5] the values of $A_{\mu}$ are presented for the case of flows of ideal and viscid gases.)

Relation (14) can be written as

$$
d \psi=\omega
$$

here $\omega=A_{\mu} d \xi^{\mu}$ is the skew-symmetrical differential form of the first degree.

Relation (15) has been obtained from the equation of the balance conservation laws for energy and linear momentum. In this relation the form $\omega$ is that of the first degree. If the equations of the balance conservation laws for angular momentum be added to the equations for energy and linear momentum, this form will be a form of the second degree. And, in combination with the equation of the balance conservation law for mass, this form will be a form of degree 3 . In general case the evolutionary relation can be written as

$$
d \psi=\omega^{p}
$$

where the form degree $p$ takes the values $p=0,1,2,3$ (The relation for $p=0$ is an analog to that in the differential forms, and it has been obtained from the interaction of energy and time).

The relation obtained from the equation of the balance conservation laws possess the properties that enable one to investigate the integrability of the original set of equations and the process of physical structures emergence.

This relation is, firstly, an evolutionary one since the original equations are evolutionary.

Secondly, it, as well as functional relation (8), turns out to be nonidentical.

To justify this we shall analyze the relation (15).

The evolutionary relation $d \psi=\omega$ is a nonidentical relation as it involves the unclosed differential form $\omega=A_{\mu} d \xi^{\mu}$.

The form $\omega$ is not a close form since its differential is nonzero. The differential $d \omega$ can be written as $K_{\alpha \beta} d \xi^{\alpha} d \xi^{\beta}$, where $K_{\alpha \beta}=\partial A_{\beta} / \partial \xi^{\alpha}-\partial A_{\alpha} / \partial \xi^{\beta}$ are the components of the differential form commutator built of the derivatives (here the term connected with the nonintegrability of the manifold has not yet been taken into account). The coefficients $A_{\mu}$ of the form $\omega$ have been obtained either from the equation of the conservation law for energy or from that for linear momentum. This means that in the first case the coefficients depend on the energetic action and in the second case they depend on the force action. In actual processes energetic and force actions have different nature and appear to be inconsistent. The commutator of the form $\omega$ made up by 
derivatives of such coefficients is nonzero. This means that the differential of the form $\omega$ is nonzero as well. Thus, the form $\omega$ proves to be unclosed and cannot be a differential like the left-hand side.

The nonidentity of the evolutionary relation (as well as the nonidentity of the functional relation (8)), points out to the fact that the conservation law equations appear to be inconsistent. This means that the initial set of equations of mathematical physics proves to be nonintegrable (it cannot be convoluted into identical relation for differentials and be integrated). In this case solutions to the initial equations of mathematical physics are not functions. They depend in addition on the commutator of the evolutionary skew-symmetric form $\omega$, which is nonzero (If the commutator be equal to zero, the evolutionary relation would be identical and this would point out to integrability of original equation).

The original equations of mathematical physics consisted of the balance conservation laws become integrable only at additional conditions when from nonidentical relation it can be obtained the identical relation (which allows to be integrated). In this case the integral structure (and hence the physical structure) is realized.

One can obtain the identical relation from nonidentical relation only when the closed exterior form will be obtained from the unclosed evolutionary form $\omega$.

But here there is some delicate matter. From the evolutionary unclosed skew-symmetric form, which differential is nonzero, one can obtain a closed exterior form with a differential being equal to zero only under degenerate transformation, namely, under a transformation that does not conserve differential (The Legendre transformation is an example of such a transformation).

Degenerate transformations can take place under additional conditions, which are caused by degrees of freedom. These conditions can be realized under a change of nonidentical evolutionary relation, which appears to be a self varying relation, since this relation is evolutionary and nonidentical.

Vanishing of functional expressions such caused as determinants, Jacobians, Poisson's brackets, residues, and others corresponds to these additional conditions. They are connected with the symmetries, which can be by the degrees of freedom (for example, the translational, rotational and oscillatory degrees of freedom of a material system).

If the conditions of degenerate transformation are realized, from the unclosed evolutionary form with nonvanishing differential $d \omega^{p} \neq 0$, one can obtain a differential form closed on pseudostructure. The differential of this form equals zero. That is, it is realized the transition

$$
d \omega^{p} \neq 0 \rightarrow(\text { degenerate transformation }) \rightarrow d_{\pi} \omega^{p}=0, d_{\pi}{ }^{*} \omega^{p}=0 .
$$

The realization of the conditions $d_{\pi}{ }^{*} \omega^{p}=0$ and $d_{\pi} \omega^{p}=0$ means that it is realized a closed dual form ${ }^{*} \omega^{p}$, which describes some structure $\pi$ (which is a pseudostructure with respect to its metric properties), and the closed exterior (inexact) form $\omega_{\pi}^{p}$, which basis is a pseudostructure, is obtained.

The realization of the closed dual form and the closed exterior (inexact) form, points out to the fact that the differential-geometrical structure, which describes the pseudostructure (closed dual form) with conservative quantity, was realized.

The physical structure, i.e. a structure on which a conservation law is fulfilled, is just such a pseudostructure with conservative quantity (The structures, from which physical fields are formatted, are such physical structures).

Thus it was obtained that the physical structures, i.e. structures on which conservation law is fulfilled, arise in the evolutionary process (described by the selfvarying evolutionary nonidentical relation) under realization of some degrees of freedom (to which a degenerate transformation is assigned).

This points out to the fact that physical strictures arise spontaneously.

Above it was investigated the evolutionary relation (15) obtained from the equations of mathematical physics when studying the consistency of the equations of balance conservation laws for energy and linear momentum. In this case one gets the differential-geometrical structures (that describe physical structures) made up by closed inexact exterior forms and corresponding dual forms of the first degree.

By a similar way it can be investigated the evolutionary relation (16) obtained from the equations of mathematical physics when studying the consistency of all equations of balance conservation laws forming the system of the mathematical physics equations (i.e., the conservation laws for energy, linear momentum, angular momentum, and mass).

From the evolutionary relation (16) one can obtain differential-geometrical structures made up by closed inexact exterior forms and relevant dual forms of the degree that takes values from 0 to 3 . This points out to the 
fact that the physical structures, which are described by these differential-geometrical structures, can be classified according the degree of closed exterior form.

The realization of the closed exterior (inexact) form $\omega_{\pi}$ points out to that on pseudostructure from evolutionary relation (15) it follows the relation

$$
d_{\pi} \psi=\omega_{\pi}
$$

which occurs to be an identical one, since the form $\omega_{\pi}$ is a differential. The identity of the relation obtained from the evolutionary relation means that on the pseudostructure the equations of conservation laws ( the equations of mathematical physics) become consistent. This points out to that on pseudostructure the equations of mathematical physics become to be integrable. This means that the physical structure (a pseudostructure with a conservative quantity) is an integral structure. The solutions of the mathematical physics equations are functions on integral structure. These are generalized solutions which are discrete functions since they are defined only on integral structures. The solutions of this type may be found by means of integrating the original equations of conservation laws for material systems.

[It should be noted a complete integrability is possible only when all balance conservation laws are consistent.]

It turns out that a pseudostructure with conservative quantity, namely, physical structure, appears to be an integrable structure. The structures like the characteristics, singular points, characteristic and potential surfaces, which are obtained when solving the mathematical physics equations, are such integrable structures.

The conditions of degenerate transformation specify the integrable structures (pseudostructures), on which the solutions become exact ones, and physical structures, on which conservation laws are fulfilled. As it was already noted, the vanishing of some functional expressions such as determinants, Jacobians, Poisson's brackets, and others corresponds to the additional conditions (which are related with degrees of freedom). For example, the characteristics are realized when the determinant becomes to be zero, the singular points arise if the Jacobian is equal to zero.

(It should be emphasized once more that the realization of differential-geometrical structure, which describes physical structure, proceeds under degenerate transformation. In this case the degenerate transformation is realized as the transition from the nonintegrable manifolds (for example, as a manifold built by the trajectories of particles or a tangent manifold of differential equations) to the integrable structures and surfaces (such as the characteristics, potential surfaces, eikonal surfaces, singular points)).

\subsection{The Physical Structures and Observable Formations}

The emergence of physical structures in evolutionary process reveals in material system as an advent of certain observable formations, which develop spontaneously. Such formations and their manifestations are fluctuations, turbulent pulsations, waves, vortices, and others.

This follows from the evolutionary relation. The evolutionary relation, which is obtained from the equation of balance conservation laws, possesses an unique property. Namely, this relation can describe the state of material medium since it contains the state functional, which specifies the material system state.

However, although the evolutionary relation includes the state functional, but, since this relation is a nonidentical one, from this relation one cannot get the differential $d \psi$ of the state functional. This points out to the absence of the state function and means that the material medium is in the non-equilibrium state.

Such non-equilibrium state is described by the solution to the mathematical physics equations, which appears to be not a function.

The non-equilibrium means that some internal forces act in material medium. It is evident that the internal force is described by the commutator of skew-symmetric form $\omega^{p}$, contained in the evolutionary relation (16). Everything that gives a contribution into the commutator of evolutionary form $\omega^{p}$ leads to emergence of internal forces that cause the non-equilibrium state of material medium (In paper [6] this problem was considered for Euler and Navier-Stokes equations).

The realization of identical relation from the evolutionary one points out to the transition of material medium to a locally-equilibrium state, since from identical relation, one can obtain the state functional, and this fact points out to the presence of state function.

The transition from non-equilibrium state to locally-equilibrium state means that nonmeasurable quantity, 
which is described by the commutator and acts as internal force, converts into a measurable quantity of material medium.

This reveals in the emergence of some observed formations in material medium. Waves, vortices, fluctuations, turbulent pulsations, and so on are examples of such formations.

Such emerged formations are described by generalized solutions to the equations of mathematical physics. The functions that correspond to generalized solutions, as it is known, are discrete functions. Such functions or their derivatives have a discontinuity in the direction normal to integrable structure [7]. Realization of integrable structures with such discontinuous functions and the transition from nonintegrable tangent manifold to integrable structure just describe the emergence of some such formations.

The intensity of such formations is controlled by a quantity accumulated by the commutator of evolutionary form (This discloses a mechanism of such processes like an origin of vortices and turbulence [5] [6]).

\section{References}

[1] Bott, R. and Tu, L.W. (1982) Differential Forms in Algebraic Topology. Springer, New York. http://dx.doi.org/10.1007/978-1-4757-3951-0

[2] Petrova, L.I. (2008) Exterior and Evolutionary Differential Forms in Mathematical Physics: Theory and Applications. Lulu.com, Morrisville, 157 p.

[3] Smirnov, V.I. (1957) A Course of Higher Mathematics. Vol. 4, Tech. Theor. Lit., Moscow. (In Russian)

[4] Clark, J.F. and Machesney, M. (1964) The Dynamics of Real Gases. Butterworths, London.

[5] Petrova, L. (2013) The Peculiarity of Numerical Solving the Euler and Navier-Stokes Equations. American Journal of Computational Mathematics, 4, 305-310.

[6] Petrova, L.I. (2014) Hidden Properties of the Navier-Stokes Equations. Double Solutions. Origination of Turbulence. Theoretical Mathematics and Applications, 4, 91-108.

[7] Petrova, L.I. (2009) Relationships between Discontinuities of Derivatives on Characteristics and Trajectories. Computational Mathematics and Modeling, 20, 367-372. http://dx.doi.org/10.1007/s10598-009-9043-0 